40 and 60), 18.9\% presented a disappointing sexual life (QS between 20 and 40) and $35.4 \%$ didn't have any partner.

Our study showed a significant correlation between the duration of the disease and the alteration of the sexual quotient, but no correlation with the clinical and biological parameters of RA.

Conclusions: We conclude that only $24.3 \%$ of our RA patients had satisfaction in their sexual life. This alteration of the sexual quotient was found to be correlated with the duration of the disease.

This fact may be explicated by the disability, fatigue and pain caused by this chronic inflammatory disease. This relation should be confirmed by future studies. References:

[1] Antonio G Tristano, Impact of rheumatoid arthritis on sexual function, World J Orthop 2014 April 18; 5(2): 107-111.

[2] Abdo J. Sex Med. 2007;4:382-389.

[3] Kraaimaat FW, et al, Intrusiveness of rheumatoid arthritis on sexuality in male and female patients living with a spouse. Arthritis Care Res 1996; 9:120-125. Disclosure of Interest: None declared

DOI: 10.1136/annrheumdis-2017-eular.5298

\section{AB0348 SLEEP DISORDERS IN PATIENTS WITH RHEUMATOID ARTHRITIS}

M. Brahem ${ }^{1}$, S. Ben Hammouda ${ }^{1}$, H. Hachfi ${ }^{1}$, M. Maraoui ${ }^{1}$, I. Haddada ${ }^{1}$

M. Jguirim ${ }^{2}$, M. Younes ${ }^{1} .{ }^{1}$ Rheumatology, Taher Sfar Hospital Mahdia, mahdia;

${ }^{2}$ Rheumatology, Fattouma Bourguiba Hospital, Monastir, Tunisia

Background: Rheumatoid arthritis (RA)as one of the most common autoimmune diseases is known to be one of the leading causes of disability.Sleep disorders have direct influence on patient's life. But the exact nature of relationship between sleep disorders and Rheumatoid arthritis is not completely understood.

Objectives: The aim of our study is to evaluate the impact of RA in sleep quality and to establish associated factors.

Methods: This is a cross-sectional and descriptive study during a period of the year 2016, including 37 patients followed in the department of Rheumatology in Mahdia Tunisia. All patients were diagnosed with RA based on ACR 1987/EULAR2010. We evaluated for each patient the parameters of activity of the disease, the quality of life by the HAQ questionnaire and the quality of sleep using two scales: Epworth (ESS) and Pittsburg scale (PSQI) which is composed from 7 components rated each one from 0 to 3 .

Results: The age of the RA patients (32 females/5 males) ranged from 21 to 76 years. The mean age was $53.1 \pm 12$ years. The mean duration of the disease was $11 \pm 10$ years [1-34]. The mean number of tender joints was $13.2 \pm 9.6$ and swollen joint was $5.9 \pm 7$. The mean DAS28 was $5.5 \pm 1.5[2.9-8.2]$ and HAQ was $1.6 \pm 0.9$ [0-2.8]. $51.3 \%$ of patients had specific joint deformations, $83.8 \%$ had radiologic involvement and $29.7 \%$ had osteoporosis.

The biologic analysis showed that the mean ESR was $45 \pm 27.1$ and the CRP was $13.7 \pm 25.3$. Rheumatoid factors were positive in $37.8 \%$ of cases, the ACPA were positive in $32.4 \%$ of cases. $81.1 \%$ of RA patients were treated by methotrexate and $13.5 \%$ were treated by biologic treatments.

The mean Epworth score was $9 \pm 5.7(0-23) .56 .8 \%$ of patients had no sleep debt, $32.4 \%$ had a sleep deficit and only $10.8 \%$ had signs of somnolence. Our study confirmed a significant correlation between the Epwoeth score and the number of tender joints, the ESR, the Health assessment quality (HAQ) score.

Regarding the overall score of Pitsburg, the average was $8.4 \pm 4.1$ (1-16). The average of the "subjective sleep quality" was 1.35 , "latency to sleep" was 1.81 , "sleep duration" was 1.24, "habitual sleep efficiency" was 1.08, "sleep disorders" was 1.62, "the use of a sleep medicine" was 0.27 and finally the average of the 7th component about "poor form during the day" was 1.11 out of 3 . So the latency to sleep and sleep disorders were the most affected components. We had a significant correlation between PSQI and the number of swollen joints, the $\mathrm{HAQ}$ score. The value of the ACPA was found to be associated with high score of PSQI.

Conclusions: Our study showed that the sleep disruption wasn't rare in patients with RA. This can be related to the disability and pain caused by this disease. Further studies with large sample size, as well as more careful tools of sleep disorders, would help to generalize results and suggestions. By providing adequate health care, and recognition of the patients' pain conditions we would ameliorate sleep quality and increase the QOL of RA patients.

References:

[1] Majid Purabdollah, et al, Relationship between Sleep Disorders, Pain and Quality of Life in Patients with Rheumatoid Arthritis; Journal of Caring Sciences, 2015, 4(3), 233-241.

[2] Seda PEHLIVAN et al, Sleep quality and factors affecting sleep in elderly patients with rheumatoid arthritis in Turkey; Turk J Med Sci (2016) 46: 1114-1121.

Disclosure of Interest: None declared

DOI: 10.1136/annrheumdis-2017-eular.5251

\section{AB0349 THE SERUM LEVEL OF IRISIN IS DECREASED IN THE PATIENTS WITH RHEUMATOID ARTHRITIS}

A.T. Kalkan ${ }^{1}$, M. Ozmen ${ }^{2}$, B. Birlik ${ }^{3}$, D. Kozaci ${ }^{4}$, I. Turkmen ${ }^{5}$, D. Solmaz ${ }^{2}$, O. Gercik ${ }^{2}$, S. Gucenmez ${ }^{2}$, S. Akar ${ }^{2} .{ }^{1}$ Department of Internal Medicine; ${ }^{2}$ Department of Internal Medicine, Division of Rheumatology; ${ }^{3}$ Department of Radyology, Izmir Katip Celebi University, School of Medicine; ${ }^{4}$ Department of Medical Biochemistry; ${ }^{5}$ Department of Musculoskeletal System and

Regenerative Medicine, Ankara Yıldırım Beyazıt University, School of Medicine, Izmir, Turkey

Background: Adipomyokines are proteins that are synthesized by and secreted from both skeletal muscle and adipose tissue, and show their effects through autocrine, paracrine or endocrine pathways (1). Irisin, a novel adipomyokine, is secreted in association with exercise from the skeletal muscle, and from the white fat tissue to help the brown fat tissue gain the energy expenditure phenotype (2). There is evidence that the irisin is associated with metabolic syndrome (MetS) and cardiovascular risk $(3,4)$.

Objectives: Rheumatoid arthritis (RA) is associated with an increased risk of cardiovascular disease (CVD) and MetS compared with the general population $(5,6)$. The aims of this study were to assess the serum level of irisin, and the possible relationships of irisin with disease activity in patients with RA.

Methods: Eighty four consecutive RA patients fulfilling the 2010 ACR/EULAR RA Classification Criteria were included in the study. Fifty age- and sexmatched healthy volunteers were enrolled as the control group. Disease duration, medications, history of traditional risk factors of CVD and demographic data of patients were noted. Body Mass Index (BMI) was calculated as "weight $(\mathrm{kg}) /$ height $(\mathrm{m})^{2}$ ". HbA1c, lipid profile, insulin were measured. Insulin resistance was assessed with the Homeostasis Model Assessment (HOMA) Index. RA disease activity was assessed by disease activity score based on evaluation of 28 joints (DAS28). Serum irisin level was assessed by ELISA. Measurement of carotid intima media thickness by carotid doppler ultrasonography was performed by a radiologist for cardiovascular risk assessment.

Results: There was no significant difference between the groups in terms of BMI $(p=0,20), \operatorname{HbA1c}(p=0,15)$, lipid profiles $(p<0.05)$, insulin resistance $(p=0.72)$ and carotid intima-media thickness $(p=0.216)$.

Serum irisin levels were found to be significantly lower in RA patients $(20,65$ (minimum:16,94- maximum:99,35) ng/mL) than healthy controls $(37,56(18,37-$ $84,70) \mathrm{ng} / \mathrm{mL})(\mathrm{p}<0.001)$. There was no relationship between RA disease activity and irisin levels.

Conclusions: This study showed that irisin was significantly lower than controls. Irisin may be responsible for increased cardiovascular risk in RA patients. But before a definite judgment; prospective studies with a larger sample size assessing the exercise behaviour of patients and the presence of CVD are necessary.

\section{References:}

[1] Raschke S, Eckel J. Adipo-myokines: two sides of the same coin - mediators of inflammation and mediators of exercise. Mediators Inflamm. 2013;320724:116.

[2] Roca-Rivada A, et al. FNDC5/irisin is not only a myokine but also an adipokine. PLoS One. 2013; 8(4):e60563.

[3] Yan B, et al. Association of serum irisin with metabolic syndrome in obese Chinese adults. PLoS One. 2014 Apr 7;9(4):e94235.

[4] Deng W. Association of Serum Irisin Concentrations with Presence and Severity of Coronary Artery Disease. Med Sci Monit. 2016 Nov 5;22:41934197.

[5] Wolfe F,et al. Increase in cardiovascular and cerebrovascular disease prevalence in rheumatoid arthritis. J Rheumatol 2003;30: 36-40.

[6] Ozmen M, et al. Prevalence of the metabolic syndrome in rheumatoid arthritis. Eur J Rheum 2014; 1: 1-4.

Disclosure of Interest: None declared

DOI: 10.1136/annrheumdis-2017-eular.2040

\section{AB0350 FUNCTIONAL DISABILITY MEASURED BY HEALTH ASSESSMENT QUESTIONNAIRE (HAQ) CORRELATES WITH DISEASE ACTIVITY IN ELDERLY RHEUMATOID ARTHRITIS PATIENTS}

N. Alpay-Kanıtez ${ }^{1}$, Ö. Pehlivan ${ }^{2}$, A. Omma ${ }^{3}$, S. Can-Sandıkçı ${ }^{3}$ S. Yılmaz-Öner ${ }^{1}$, C. Bes ${ }^{1}$, S. Çelik ${ }^{1}$. ${ }^{1}$ Bakırköy Dr. Sadi Konuk' Research and Training Hospital; ${ }^{2}$ Umraniye Research and Training Hospital, Istanbil; ${ }^{3}$ Ankara Numune Research and Training Hospital, Ankara, Turkey

Background: Rheumatoid arthritis (RA)'s prevalence increases with age and the recognition of functional disability related to RA could be challenging in elderly patients (1).

Objectives: In this study, we aimed to look at the correlation between disease activity and the functional disability by using HAQ score in elderly RA patients.

Methods: Elderly RA patients, $\geq 65$ years old at their routine visits were included to the study. The composite "disease activity score" in 28 joints (DAS-28) and "Physician Global Assessment" (PhGA) were used to determine disease activity. Health assessment questionnaire (HAQ) scores were calculated to describe the functional disability and compared across the disease activity groups according to DAS-28.

Results: Two hundred and fifty eight RA patients with the mean age of $71 \pm 5$ 
(65-90) were included. Seropositivity rate was $71 \%$ and $47 \%$ of the patients had erosive disease. Joint deformity was seen in $72(28 \%)$ of patients and $28(11 \%)$ patients had undergone total arthroplasty. Lung involvement (8.5\%) and Sjogren's syndrome $(7.4 \%)$ were found as the most frequent extra-articular manifestations. Hypertension (66\%) and osteoporosis $(36 \%)$ were the common co-morbidities. The proportion of patients with high and moderate disease activity was found as $70 \%$. HAQ and PhGA scores were significantly correlated with the disease activity (Figure).
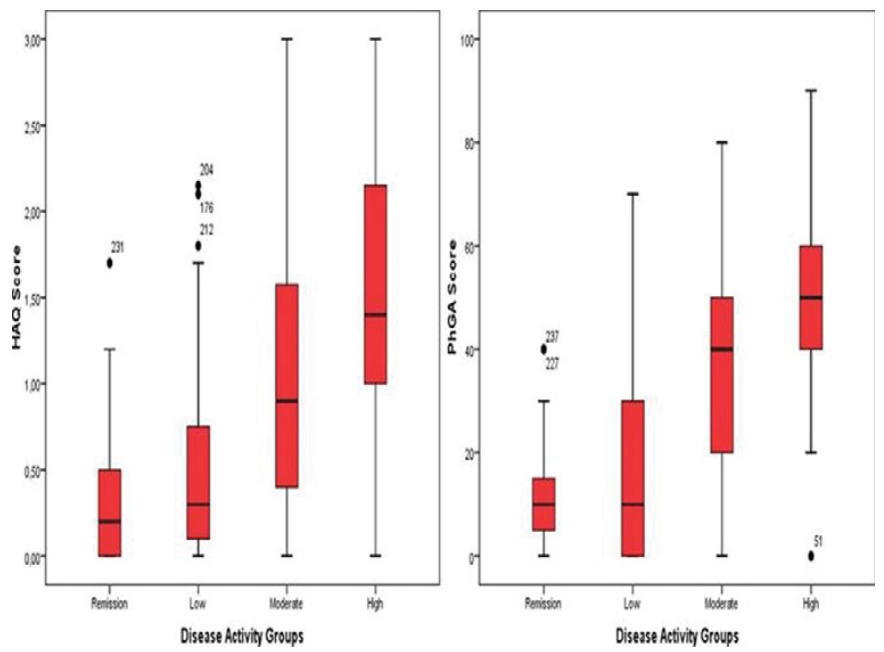

Figure 1. HAQ and PhGA distribution in disease activity subgroups. Both of them were found correlated with disease activity (p: 0.000).

Conclusions: We provided a novel data concerning the usefulness of $H A Q$ for prediction of disease activity in RA patients who are elderly as well. In the future, our report will be supported by the studies which suggest that HAQ score improve with effective treatment in elderly RA patients.

References:

[1] Uhlig T, Loge JH, Kristiansen IS, Kvien TK. Quantification of reduced healthrelated quality of life in patients with rheumatoid arthritis compared to the general population. J Rheumatol 2007;34: 1241-1247.

Disclosure of Interest: None declared

DOI: 10.1136/annrheumdis-2017-eular.6662

\section{AB0351 CORRELATION BETWEEN SERUM ANTI-CITRULLINATED PROTEIN ANTIBODIES AND SUBCLINICAL CARDIC AFFECTION IN RHEUMATOID ARTHRITIS PATIENTS}

A. Helal ${ }^{1}$, G. Younis ${ }^{1}$, N. Hussein ${ }^{1}$, E. Elsharkawy ${ }^{2}$,

N. Abdelhady Abdelsadek ${ }^{1}$. ${ }^{1}$ Physical Medicine, Rheumatology and

Rehabilitation, Alexandria University Hospital; ${ }^{2}$ Cardiology and Angiology, Faculty of Medicine, Alexandria, Egypt

Background: Cardiovascular disease (CVD) is a major cause of morbidity and mortality in rheumatoid arthritis (RA) patients. It has been postulated that chronic inflammatory activity is important for the development of CVD in RA even after adjustment for traditional cardiovascular risk factors. ${ }^{(1)}$ One of the changes occurring in the context of inflammation is citrullination. Development of anti-citrullinated protein antibodies (ACPA) is implicated in higher frequency of extra-articular manifestations including cardiovascular complications. ${ }^{(2)}$

Objectives: To assess the relation of ACPA to subclinical cardiac affection in RA patients.

Methods: Thirty RA patients fulfilling the 2010 ACR-EULAR classification criteria for RA with no clinically evident CVD were subjected to full history taking and clinical examination. Disease activity was assessed by 28 -joint disease activity score based on C-reactive protein (DAS28-CRP) (4 variables). The levels of ACPA, CRP, total cholesterol, triglycerides, high density lipoprotein cholesterol and low density lipoprotein cholesterol were measured. The patients were subjected to M-mode and colour Doppler echocardiographic examination.

Patients were subdivided into two subgroups according to ACPA positivity (ACPA positive patients represented "group A" and ACPA negative patients represented "group B").

Results: The frequency of subclinical cardiac affection by echocardiographic examination was significantly higher among group A patients (4 patients had valvular lesion and 9 patients had diastolic dysfunction) than in group B patients (3 patients had diastolic dysfunction), $(p=0.011)$. ACPA level showed significant positive correlation with isovolumic relaxation time (IVRT) in group A patients (prolongation of IVRT is a sign of diastolic dysfunction), $(p=<0.001)$

Conclusions: The presence of ACPA is related to development of subclinical cardiac involvement in RA patients and all RA patients with high level of ACPA should be routinely evaluated with echocardiography to assess their cardiovascular status.
Comparison between the two studied groups according to subclinical cardiac affection

\begin{tabular}{|c|c|c|c|c|c|c|}
\hline & \multicolumn{2}{|c|}{$\begin{array}{c}\text { Group A } \\
(n=18)\end{array}$} & \multicolumn{2}{|c|}{$\begin{array}{c}\text { Group B } \\
(n=12)\end{array}$} & \multirow[t]{2}{*}{ Test of sig. } & \multirow[t]{2}{*}{$p$} \\
\hline & No. & $\%$ & No. & $\%$ & & \\
\hline \multicolumn{7}{|c|}{ Subclinical cardiac affection } \\
\hline Present & 13 & 72.2 & 3 & 25.0 & $\mathrm{FE}^{2}{ }^{2}=6.451$ & $0.011^{*}$ \\
\hline Absent & 5 & 27.8 & 9 & 75.0 & & \\
\hline
\end{tabular}

FE: Fisher Exact for Chi square test, $x^{2}$ : Chi square test. ${ }^{*}$ Statistically significant at $p \leq 0.05$.

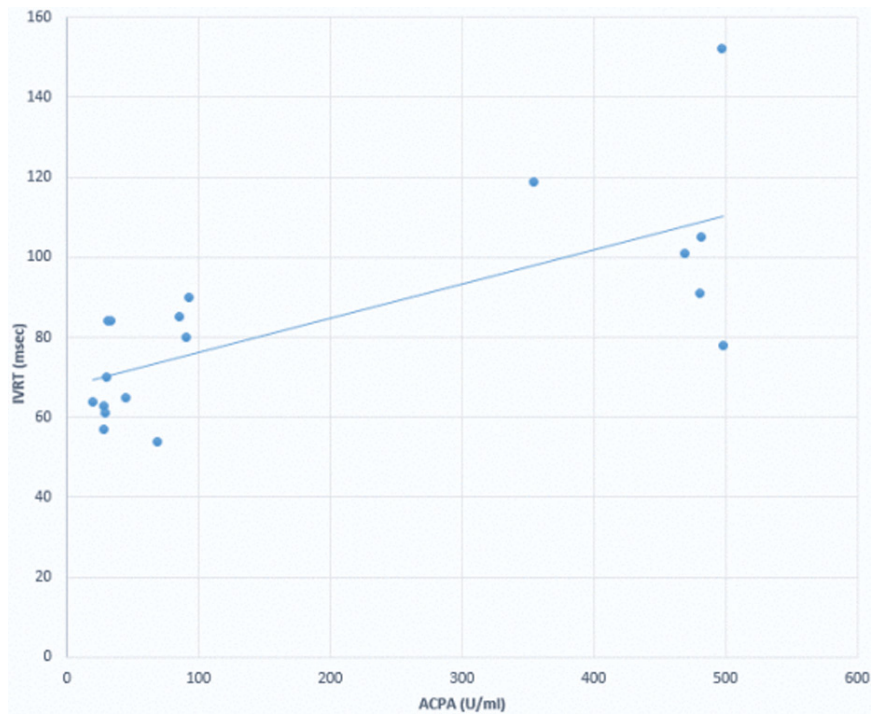

References:

[1] Arnab B, Biswadip G, Arindam P, Shyamash M, Anirban G, Rajan P. Anti-CCP antibody in patients with established rheumatoid arthritis: Does it predict adverse cardiovascular profile? J Cardiovasc Dis 2013; 4(2):102-6.

[2] Crowson C, Liao K, Davis J, Solomon D, Matteson E, Knutson K, et al. Rheumatoid arthritis and cardiovascular disease. Am Heart J 2013; 166(4):622-28.

Disclosure of Interest: None declared

DOI: 10.1136/annrheumdis-2017-eular.2801

\section{AB0352 RISK FACTORS AND SUBCLINICAL ATHEROSCLEROSIS IN RHEUMATOID ARTHRITIS FEMALES COMORBID WITH HYPERTENSION}

O. Kuryta, T. Lusynets, O. Sirenko. State Establishment "Dnipropetrovsk medical academy of Health Ministry of Ukraine", Dnipropetrovsk, Ukraine

Background: Rheumatoid arthritis (RA) associates with accelerated atherosclerosis and high cardiovascular mortality. Cardiovascular risk management in RA comorbid with HT pts is do not fully reflected by traditional risk scales, thus additional factors searching is required.

Objectives: We aimed to estimate the subclinical manifestations of atherosclerosis in RA females comorbid with HT and its relationship with traditional, additional risk factors.

Methods: The study included 112 RA females with comorbid HT (mean age of 54 $[50,3 ; 61,5]$ years) and $105 R A$ females without $H T$ (control group). All pts received stable therapy of RA more than 6 months. Pts with coronary artery disease were excluded. The risk of fatal cardiovascular disease was calculated using $\mathrm{m}$ SCORE (EULAR 2010). RA disese activity was measured using DAS28 scale. Carotid ultrasound with stiffness indexes detection and endothelial-dependent flow mediated vasodilatation (EDVD) by Celermajer method were performed. The levels of adiponectin, insulin were measured using ELISA kit test, insulin resistance was estimated using HOMA2 index.

Results: Subclinical manifestations of atherosclerosis were established in 88 (78.6\%) RA females with HT and $55(52,4 \%)$ control group pts. The majority of main group pts have atherosclerotic plaques - 62 (55.4\%), unstable plaques had $26(23.2 \%)$ pts. While only $33(29,5 \%)$ patients were high and very high cardiovascular risk assessed by mSCORE. In compare, 35 (33,3\%) control group pts have atherosclerotic plaques, unstable plaques had only $14(13.3 \%)$ control pts $(p<0.05)$. The presence of atherosclerotic plaques in RA females with HT was associated with age, RA disease activity, endothelial dysfunction, carotid stiffness, LDL cholesterol level, insulin resistance, adiponectin level, duration of steroid therapy. AUROC index for adiponectin and HOMA2 were $0.83(95 \% \mathrm{Cl} 0.74-0.95$; $\mathrm{p}<0.05$ and $0.75(95 \% \mathrm{Cl} 0.68-0.91 ; \mathrm{p}<0.05$ respectively, that indicate a good quality of diagnostic models.

Conclusions: RA females with comorbid HT are characterized by higher frequency of subclinical atherosclerosis with unstable atherosclerotic plaques in compare with controls. Endothelial function, insulin resistance, adiponectin level, 